\title{
Anestesia espinal: Parte I. Historia
}

\section{Spinal anesthesia: Part I. History}

Juan Carlos De La Cuadra F. ${ }^{1}$, Fernando Altermatt C. ${ }^{1}$, Catalina Kychenthal L. ${ }^{2}$, María Jesús Irarrázaval M. ${ }^{2}$, Héctor Lacassie Q. ${ }^{1}$

\begin{abstract}
Spinal anesthesia is a fundamental type of regional anesthesia, very frequently used in clinical practice. In order to achieve the safe and widely known technique we use today, multiple events have happened throughout history. Numerous advances in anatomy, physiology, pharmacology, needle development and others, gave way to the first spinal anesthesia in 1898 and all the progress that has hence been made. Multiple discoveries and adaptations have been made since that moment, finally reaching the technique that is known and used nowadays.
\end{abstract}

\section{RESUMEN}

La anestesia espinal es una forma de anestesia regional fundamental en la práctica clínica. Para llegar a la técnica segura y ampliamente difundida que hoy se conoce, han sucedido diversos eventos a lo largo de la historia. Numerosos avances en cuanto a conocimiento anatómico, fisiología, farmacología, desarrollo de agujas y otros, dieron paso a la primera anestesia espinal en 1898 y a todo el avance que desde entonces se ha logrado. Múltiples descubrimientos y adaptaciones se han hecho desde entonces, alcanzando finalmente la técnica utilizada por anestesistas hoy en día.

\section{Key words:}

Anesthesia,

regional anesthesia, spinal anesthesia, history of medicine

\section{Palabras clave:}

Anestesia, anestesia regional, anestesia espinal, historia de la medicina

1 División de Anestesiología, Escuela de Medicina, Pontificia Universidad Católica de Chile. Santiago, Chile.

2 Escuela de Medicina, Pontificia Universidad Católica de Chile. Santiago, Chile.

Fecha de ingreso: 12 de octubre de 2020

Fecha de aceptación: 15 de octubre de 2020

\section{ORCID}

0000-0002-0464-8643

Sin fuentes de financiamiento

No se declaran conflictos de interés

Correspondencia:

Fernando Altermatt Couratier

falterma@med.puc.cl 


\section{Introducción}

L a anestesia espinal es una herramienta fundamental en la práctica anestesiológica. Es considerada muchas veces como sinónimo de anestesia regional, aunque ésta claramente abarca otras técnicas neuraxiales y periféricas. Una encuesta de la práctica en Chile en el año 2010 encontró que 98\% de los anestesiólogos que respondieron, usaban anestesia subaracnoídea[1]. Aunque la primera anestesia regional descrita fue tópica y luego local infiltrativa, podemos decir que la anestesia espinal es la primera técnica publicada de inyección percutánea de anestésico local perineural. Desde sus inicios es vista como una práctica más segura que la narcosis con cloroformo y éter, disminuyendo la mortalidad, estados de shock y los efectos adversos postoperatorios[2]. A pesar de las ventajas clínicas también tiene sus propios riesgos: desde su primera publicación se identifica la aparición de la conocida cefalea post punción y el riesgo de daño neurológico, que es el gran temor que ahuyenta a médicos y pacientes.

En una serie de artículos revisaremos distintos aspectos de la anestesia espinal. En esta primera publicación se presenta su historia.

\section{Historia de la anestesia espinal}

\section{La primera anestesia}

La primera descripción de una anestesia espinal planificada como tal fue realizada por el cirujano alemán August Bier en agosto de 1898 para manejo quirúrgico de una lesión de tobillo. En su publicación de 1899 presenta los resultados de seis pacientes además de la experiencia aplicada en él mismo y su ayudante[3].

En este relato ya se describe el curso diferenciado del bloqueo de distintas sensibilidades y motor: "Con la inyección hubo sensación de calor; a los 7 minutos, pinchazos en el muslo y cosquillas en las plantas de los pies son apenas percibidos; a los 8 minutos, una pequeña incisión en el muslo y la introducción de una aguja hasta el fémur no causan dolor sino que solo sensación de presión"[3]. También describe trastornos clínicos que hoy suponemos asociados a cambios hemodinámicos y más de $50 \%$ de cefalea post procedimiento. La cefalea fue descrita en estos términos: "a las 15 horas presentó cefalea intensa y mareos, especialmente, al levantarse; todos sus síntomas se reproducían al levantarse y desaparecían al acostar$\mathrm{se}^{\prime \prime}[3]$.
Para llegar a este punto inicial, han debido suceder varios otros descubrimientos y desarrollos. Elementos que ayer, hoy y mañana seguirán siendo fundamentos en el conocimiento y práctica de esta técnica: anatomía, fisiología, farmacología, desarrollo de agujas y otros elementos técnicos.

\section{Antecedentes anatómicos}

Antonio Valsalva (1666-1723) en sus estudios de anatomía hace notar la presencia de un líquido claro al disecar la columna[4]. En 1764 Dominico Cotugno y en 1825 François Magendie profundizaron el estudio y describieron en forma mas completa el líquido cefalorraquídeo[3]. Heinrich Quincke realizó las primeras punciones lumbares con aguja en 1891 para tratar patologías con hidrocefalia. En su reporte de 1892 hace referencia a Magendie y como éste cita a Cotougn[4]. August Bier estudió y trabajó en la Universidad de Kiel en Alemania, donde compartió con Quincke y von Esmarch[5]. Bier probablemente usó una aguja diseñada por Quincke en sus anestesias.

En 1885, el neurólogo estadounidense James L. Corning en un intento de tratar afecciones neurológicas, inyecta cocaína por un abordaje intervertebral lumbar bajo en un perro y luego en un paciente[6]. Corning esperaba que el anestésico fuese absorbido por el flujo sanguíneo local y llevado a la superficie de la medula espinal. El resultado fue una parálisis motora y sensitiva de las extremidades traseras en el perro, mientras que en el paciente, disminución de sensibilidad en extremidades inferiores, región lumbar, pene y escroto, sin alteración motora evidente.

Algunos han considerado este episodio como la primera anestesia espinal. El hecho de que nunca se describió liquido cefalorraquídeo, además de la gran masa de cocaína usada para obtener los efectos descritos, sugieren que ésta no fue una anestesia espinal, sino probablemente una epidural[6].

Quizás Corning no realizó una inyección subaracnoidea de anestésico local en sus primeras experiencias, ni buscaba proveer anestesia quirúrgica, pero fue quien primero acuñó el término de "anestesia espinal" y vio su potencial uso en esta área.

\section{Antecedentes farmacológicos}

La coca es un arbusto que se da en el área amazónica de Sudamérica. El médico peruano Hipólito Unane (1755-1833) describió el efecto de la coca como: "restaurador y antifatigante, disipador de obstrucciones, cura los cólicos y modera o extingue los dolores tópicos"[7]. La hoja de coca fue llevada 
a Europa donde el interés por la cocaína se centraría en su capacidad de aumentar el rendimiento físico y mental y su capacidad para mitigar el hambre. Aunque hay sugerencias del uso de la planta de coca por indígenas como anestésico local, en la cultura occidental la primera referencia del efecto anestésico de la hoja de coca fue escrita por el jesuita Bernabé Cobo en 1653[8]. En Europa, solo a mediados del siglo 19 aparecen comentarios sobre este aspecto. Albert Niemann, químico alemán, logró aislar el compuesto activo en 1860 y fue quien dio el nombre de cocaína[8].

En septiembre de 1884, Carl Koller, oftalmólogo austríaco, da cuenta ante la Sociedad de Oftalmología de Alemania, la posibilidad de dar anestesia quirúrgica de conjuntiva y córnea con gotas de cocaína[9]. Este hecho se considera el inicio de la anestesia regional. En los meses siguientes se había ensayado el uso de cocaína en distintos lugares de Alemania e incluso en Estados Unidos. Del uso en mucosa y conjuntivas comenzó a ensayarse el efecto anestésico con cocaína en otros territorios del cuerpo.

\section{De las agujas}

El desarrollo de agujas para inyección probablemente va asociado a la evolución de las cánulas de drenaje y de jeringas. Desde la antigüedad el hombre aprendió a usar elementos que encontraba en la naturaleza como el cálamo de una pluma, cañas vegetales e incluso huesos pequeños y largos para drenar colecciones y luego para desobstruir vejigas. El manejo de los metales generó instrumentos que cumplieron estos usos. Con el tiempo su pudo variar formas, grosores y largos[10]. Las primera jeringa y aguja de inyección hipodérmica es disputada entre Rynd, Pravaz y Wood[10]. Corning durante sus estudios desarrolló su propia aguja espinal. Esta era flexible y con una punta biselada corta, replicando la aguja hipodérmica de Wood. Quincke en su trabajo en punciones lumbares también desarrolló su propia aguja, descrita con una punta biselada cortante larga y fue la que probablemente usó Bier. El uso de un mandril o estilete era usado para limpiar las agujas entre usos, producto del óxido con los lavados. En el desarrollo de agujas espinales, estos estiletes eran necesarios para impedir que se obstruyera la aguja con tejido durante su avance y evitar llevar piel y u otro material hacia el raquis[11].

\section{Después de la primera anestesia espinal}

Luego de la publicación de Bier en 1899, numerosos cirujanos publicaron sus experiencias. Destacable es otro cirujano, el francés Théodore Tuffier, quien haría demostraciones de inyección espinal de cocaína en el Congreso Médico Internacional de 1900 en Paris. A partir de este congreso se difunde aún más su práctica. En España, la primera serie, que comprende 16 pacientes, se publica en 1900 por el cirujano catalán Francisco Rusca[12]. En el continente americano, el ensayo de esta nueva técnica también aparece precozmente luego de la experiencia europea. En Estados Unidos, en menos de dos semanas se realizan las primeras experiencias. En San Francisco, dos cirujanos, Dudley Tait y Guido Calieri la realizan el 26 octubre de 1899 y en Nueva Orleáns, Rudolph Matas el 10 de noviembre del mismo año[13].

En Latinoamérica, en menos de un año se han realizado también las primeras experiencias. El Dr. Ramón Pardo Galíndez, médico del estado de Oaxaca, fue el primero en realizar una raquianestesia en la República Mexicana, en julio de 1900[14]. En Buenos Aires, Argentina, José Sabatini ensaya la cocainización raquídea no quirúrgica el 28 de abril de 1900. En 1901, en Buenos Aires, Bartolomé Podestá publica su tesis: "Las inyecciones de cocaína en la aracnoides lumbar". Contaba en ese entonces con 195 anestesias raquídeas, sin ninguna muerte.

En las revisiones y estudios históricos realizadas por el Dr. Samuel Barros, anestesiólogo e historiador chileno, nos da cuenta de la primera anestesia espinal en Chile y según él, en Latinoamérica. El Dr. Alcibíades Vicencio, gíneco obstetra chileno (1859-1913), primer director de la Escuela de Obstetricia para Matronas de la Universidad de Chile, (cuyo nombre llevaría años más tarde esta institución que él dirigiera por 26 años), fue el primero en administrar una anestesia raquídea en Chile, que fue para analgesia del trabajo de parto. La hizo el 15 de junio de 1900, apenas una semana después de la que se considera la primera anestesia/analgesia raquídea para trabajo de parto en el mundo, que fue realizada por Oskar Kreiss (gíneco obstetra suizo). Su experiencia la presentó oficialmente en la reunión mensual de la Asociación Médica en Santiago en julio de 1900[15].

En 1904, la síntesis de estovaína y posteriormente procaína en 1905, valoriza la técnica luego de los problemas por toxicidad de la cocaína y los accidentes atribuidos a ella. La procaína se convirtió en el anestésico local de elección por su baja toxicidad. Su limitación era la corta duración, pero la asociación con adrenalina lograba prolongar el efecto. La spinocaína fue un preparado de procaína con alcohol y estricnina, una solución hipobárica que permitía modificar el nivel de la anestesia[16]. La tetracaína se comenzó a usar en 1929, incorporando la posibilidad de anestesia espinal de larga duración[17]. En 1949, aparece en 
el mercado la lidocaína. Esto significó un gran cambio y la vuelta a muchas técnicas regionales que se habían abandonado por falta de drogas que dieran entera satisfacción. Desde entonces la familia de las aminoamidas han dominado entre los anestésicos locales.

Durante los primeros años del siglo XX, ya se habían descrito mucho de los efectos adversos y algunas complicaciones neurológicas asociadas a la técnica. Se describió el uso de adrenalina para prolongar el efecto de los anestésicos y la adición de glucosa para controlar el nivel de bloqueo. La excelente calidad anestésica otorgada por la técnica, frente a la anestesia general de esta primera mitad del siglo, favoreció su frecuente uso.

Tempranamente y en forma correcta se postuló que la cefalea post anestesia espinal se debía al orificio en las membranas del saco dural, que a su vez dependía del tamaño y característica de la punta de la aguja utilizada. El desarrollo de materiales y la industria permitieron el diseño de nuevas agujas más finas y con nuevas puntas. En 1926, se publica una serie de 215 pacientes con sólo 2 cefaleas al usar una nueva aguja de punta redonda pero aguzada, la primera aguja Ilamada "atraumática"[11]. Estudios actuales han demostrado que estas agujas punta de lápiz precisamente por ser mas traumatizantes del tejido es que se traducen en menos cefaleas[18].

No podemos dejar de hacer una referencia a las agujas de Tuohy. Edward B. Tuohy fue un prestigioso anestesiólogo estadounidense, quien llegara a ser presidente de la Sociedad Americana de Anestesiólogos (ASA). Estaba atraído por la anestesia espinal continua. La aguja habitual para esta técnica (aguja de Barker) tenía punta en bisel. Tuohy decidió reemplazar ésta por una aguja con una punta direccionada, diseñada por el dentista Ralph Huber. Tuohy agregó a esta aguja un estilete, para evitar que el lumen se obstruyera con tejido durante su avance[19]. Pero fue el anestesiólogo cubano Manuel Martínez Curbelo quien en 1949 por primera vez publica el uso de una técnica peridural lumbar continua en Estados Unidos, usando la aguja de Tuohy[20].

En la segunda mitad del siglo $X X$, suceden algunos hechos relevantes que implican un creciente abandono de la anestesia espinal. En 1942, la introducción del curare en la práctica clínica de la anestesia general, precursor de los relajantes musculares ampliamente utilizados hoy en día y posteriormente, la síntesis de nuevos anestésicos inhalatorios, mejoran la calidad de la anestesia general. Por otro lado, aparece el caso de dos pacientes en Inglaterra con daño neurológico post anestesia espinal[21]. Fue conocido como el caso Woolley y Roe en 1947, dos hombres jóvenes, el mismo día, en el mismo hospital y por el mismo anestesista, fueron sometidos a anestesia espinal, resultando con lesiones neurológicas graves y permanentes. El proceso judicial posterior declaró inocente al anestesiólogo. El juez finalmente aceptó la teoría de contaminación por fenol del anestésico durante su proceso de asepsia. Este caso genera en el ámbito médico, sin embargo, el temor por la judicialización de los eventos de accidentes y daño en los pacientes. En este contexto se tiene la sensación de que si el paciente fallecía durante una anestesia general, el forense sentenciaría que él no "resistió" la anestesia y todos estarían conformes con una muerte accidental, pero un paciente parapléjico post anestesia espinal, significaría un largo juicio y/o un cuantioso arreglo económico[21)].

La anestesia espinal vuelve a resurgir alrededor de la década de 1970. Nuevos anestésicos locales, la incorporación definitiva de las agujas punta lápiz, el mejor entendimiento de la fisiología de la anestesia espinal, de sus efectos adversos y su manejo, finalmente han reposicionado esta técnica como segura, con grandes beneficios y una importante alternativa en la anestesia moderna. La anestesia espinal es conocida también como anestesia subaracnoidea, anestesia intratecal, anestesia raquídea o raquianestesia.

\section{Referencias}

1. Corvetto MA, Carmona J, Vásquez MI, Salgueiro C, Crostón J, Sosa R, et al. Práctica actual de la anestesia regional en América del Sur: encuesta en línea. Rev Esp Anestesiol Reanim. 2017;141-6. https://doi. org/10.1016/j.redar.2016.05.001

2. Allen C. Local and Regional Anesthesia. 2nd ed. Philadelphia: W. B. Saunders; 1918.

3. Wulf HFW. The centennial of spinal anesthesia. Anesthesiology. 1998;500-6. https:// doi.org/10.1097/00000542199808000-00028
4. Vandam LD. On the origins of intrathecal anesthesia. Reg Anesth Pain Med. 1998;335-9. https:// doi.org/10.1097/00115550199823040-00002

5. Van Zundert A, Goerig M. August bier 1861-1949 a tribute to a great surgeon who contributed much to the development of 
modern anesthesia on the 50th anniversary of his death. Reg Anesth Pain Med. 2000;26-33. https://doi.org/10.1016/\$10987339(00)80007-3

6. Marx GF. The first spinal anesthesia who deserves the laurels? Reg Anesth. 1994;42930.

7. Dagnino Sepúlveda J. De la coca a la cocaína. ARS MEDICA Rev Ciencias Médicas. 2016;280-91. https://doi.org/10.11565/arsmed. v32i1.270

8. Calatayud J, González Á. History of the development and evolution of local anesthesia since the coca leaf. Anesthesiology. 2003. p. 1503-8. https:// doi.org/10.1097/00000542200306000-00031

9. Jorge Dagnino S. El trío de viena. De la cocaína a la anestesia tópica. Rev Chil Anest. 2011;24-40.

10. Kirkup J. Surgical history: The history and evolution of surgical instruments: VIII: Catheters, hollow needles and other tubular instruments. Annals of the Royal College of Surgeons of England. 1998. p. 81-90.

11. Calthorpe N. The history of spinal needles: Getting to the point. Anaesthesia. 2004;1231-41. https://doi.org/10.1111/j.13652044.2004.03976.x

12. Hervás C, Cahisa M. Centenario de la raquianestesia en Espana: Los primeros pasos (1899-1904). Revista Espanola de Anestesiologia y Reanimacion. 2000. p. 216-21.

13. Larson MD. Tait and Caglier: The first spinal anesthetic in America. Anesthesiology. 1996;85(4):913-9. https:// doi.org/10.1097/00000542199610000-00028

14. Marrón-Peña GM. Historia de la anestesia gineco-obstétrica en México. Revista Mexicana de Anestesiologia. 2013. p. 212-8.

15. Barros S. First Spinal Anesthesia for Obstetrics in the American Continent. In: Anesthesia History Association 15th Annual Spring Meeting [Internet]. Augusta, Georgia; 2009. p. 7. Available from: http:// newsite.ahahq.com/wp-content/uploads/2018/05/2009AHA-Annual-Spring-Meeting. pdf

16. Ball C, Westhorpe R. Local anaesthetics - Procaine (Novocaine, ethocaine). Anaesthesia and Intensive Care. 2004. p. 303. https://doi.g/10.1177/031005

\section{X0403200301}

17. Ball C, Westhorpe R. Local anaesthetics - Nupercaine and Amethocaine. Anaesthesia and Intensive Care. 2004. p. 457. https://doi.g/10.1177/031005 7X0403200401

18. Reina MA, De León-Casasola OA, López A, De Andres J, Martin S, Mora M. An in vitro study of dural lesions produced by 25-gauge Quincke and Whitacre needles evaluated by scanning electron microscopy. Reg Anesth Pain Med. 2000;393-402. https:// doi.org/10.1097/00115550200007000-00013

19. Frölich MA, Caton D. Pioneers in epidural needle design. Anesth Analg. 2001;215-20. https:// doi.org/10.1097/00000539200107000-00043

20. Curbelo MM. Continuous peridural segmental anesthesia by means of a ureteral catheter. Curr Res Anesth Analg. 1949;1-4. https:// doi.org/10.1213/00000539194901000-00002

21. Maltby JR, Hutter CDD, Clayton KC. The Woolley and Roe case. Br J Anaesth. 2000;121-6. https://doi.org/10.1093/oxfordjournals.bja.a013370 